\title{
ALGUNOS EFECTOS DE LA SALINIDAD EN EL CULTIVO DEL TOMATE Y PRÁCTICAS AGRONÓMICAS DE SU MANEJO ${ }^{1}$
}

\author{
SOME EFFECTS OF SALINITY ON THE TOMATO CULTIVARS AND \\ AGRONOMIC PRACTICES IN ITS MANAGING
}

\author{
Vitelio Goykovic Cortés ${ }^{2}$; Gabriel Saavedra del Real
}

\begin{abstract}
RESUMEN
La salinidad afecta de diversas maneras a las plantas de tomate. La mayoría de los efectos son adversos. Por ejemplo, el porcentaje de germinación disminuye y se prolonga el tiempo en el cual las semillas llevan a cabo este proceso. A nivel de raíces, éstas alcanzan una menor longitud de modo que el volumen de suelo que prospectan es menor.

Los órganos del sistema aéreo también se alteran por efecto de las sales, los tallos alcanzan una menor altura, las hojas se reducen en número y presentan desecación en sus bordes de modo que hay menos producción de fotoasimilados. El número y peso de los frutos también se afectan negativamente de manera que su rendimiento comercial disminuye.

En las especies silvestres de tomate y en los cultivares se ha detectado variabilidad en la respuesta a salinidad, siendo algunas más tolerantes que otras, de modo que éstas pueden utilizarse como fuente de genes para su mejoramiento.

La salinidad puede mejorar la calidad de los frutos en términos organolépticos y biológicos, al presentar éstos un mayor contenido de compuestos solubles, concentración de ácidos y licopeno.

Existen un sinnúmero de prácticas de manejo que pueden ayudar a mejorar la producción de tomates en condiciones salinas como, por ejemplo, el injerto, mejoramiento del drenaje, fertilización potásica y cálcica e inoculación del suelo con micorrizas, entre otras.

Palabras clave: Tomates, Lycopersicon esculentum, salinidad, prácticas de manejo.
\end{abstract}

\begin{abstract}
Salinity affects the tomato plants in positive and negative terms. The majority are adverse effects. For example, the percentage of germination diminishes and the time in which this process is carried out extends The roots are shorter so the soil volume for exploring is smaller.

The aerial system is also altered, the stems have shorter height, the leaves are reduced in number and present dry edges; therefore, there is less production of photoassimilates. The number and weight of the fruits are also negatively affected, so their commercial value diminishes.

In the wild species of tomatoes there has been detected variability in some responses to salinity, some of them presenting more tolerance than others. The salinity improves the quality of the fruits in organoleptic and biological terms, presenting these a greater content of soluble solids and lycopene, and acids concentration.

There are a number of management practices that can help to improve the production of tomatoes under saline conditions such as the grafting, drainage improvement, potassium and calcium fertilization, and soil inoculation with mycorrhizas among others.
\end{abstract}

Key words: Tomatoes, Lycopersicon esculentum, salinity, management practices.

\section{INTRODUCCIÓN}

En muchas áreas del mundo dedicadas a la agricultura la obtención de buenos rendimientos, así como también el poder cultivar una amplia variedad de especies, cada vez está teniendo más restricciones debido a la salinización de los suelos. Se estima que sobre 800 millones de hectáreas en

\footnotetext{
1 Este trabajo contó con el patrocinio del Centro de Investigaciones del Hombre en el Desierto (CIHDE).

2 Facultad de Ciencias Agronómicas, Universidad de Tarapacá, Casilla 6-D. E-mail: vgoykovi@uta.cl

3 (INIA) Instituto de Investigaciones Agropecuarias La Platina, Santiago. E-mail: gsaavedr@inia.cl
} 
el planeta están afectadas por sales, de estas 397 millones lo son por problemas de salinidad y 434 millones por condiciones asociadas a sodicidad (Munns, 2005; FAO, 2000). Varias son las causas vinculadas a estos procesos de salinización, entre las cuales es posible citar un excesivo empleo de fertilizantes, uso de agua de mala calidad por el exceso de sales, mal drenaje y tala de vegetación arbórea (Tanwar, 2003).

El tomate (Lycopersicon esculentum Mill) cuando se cultiva en suelos salinos no se encuentra ajeno a una disminución de los rendimientos, puesto que es una especie glicófita, medianamente sensible a las sales y que presenta un umbral respecto al contenido total de sales, cuantificadas en el extracto de saturación del suelo y expresadas como conductividad eléctrica (CEs) de 2,5 dS/m (Chinnusamy et al., 2005). A nivel mundial, es una hortaliza importante; el año 2002 se produjeron en el mundo 120.684.961,8 toneladas, participando Chile con 1.341.268,5 toneladas (USDA, 2003).

El cultivo del tomate en áreas con problemas de salinidad provoca en las plantas un sinnúmero de efectos fisiológicos, morfológicos y bioquímicos, tales como disminución de la fotosíntesis (Singh y Chatrath, 2001), un menor peso de los frutos (Del Rosario et al., 1990; Pérez-Alfocea et al., 1996) y cambios cuantitativos y cualitativos en la síntesis de proteínas por cambios en la expresión de genes a causa de la salinidad, entre otros (Singh y Chatrath, 2001).

Al examinar los efectos de las sales en la germinación como en los órganos de las plantas de tomates, la gran mayoría son adversos, pocos presentan un carácter positivo. A nivel de germinación, a medida que aumenta la concentración de sales en el medio, el porcentaje de germinación disminuye y el periodo en que este proceso se lleva a cabo se prolonga (Cuartero y Fernández-Muñoz, 1999; El-Habbasha et al., 1996; Singer-SM, 1994; Foolad y Lin, 1997). Estas respuestas se observan tanto en la especie cultivada como en las silvestres. A pesar de la poca variabilidad genética que se le atribuye al tomate cultivado (Nuez, 2001) se han detectado algunos cultivares con mayor tolerancia, como el cv Edkawy. En los tomates silvestres, de gran interés para el mejoramiento genético de la especie cultivada, en las especies Lycopersicon chilense, L. peruvianum, L. pennellii, L. cheesmanii y $L$. pimpinellifolium también se han detectado accesiones con tolerancia a salinidad (Rick, 1982).

A nivel de raíces, las sales alteran la absorción de agua afectando el crecimiento de estos órganos; también actúan produciendo efectos tóxicos. $\mathrm{La}$ magnitud de las respuestas de las plantas se encuentra estrechamente relacionada a la concentración de las sales, a la duración del estrés a que están expuestas y a la especie o cultivar de que se trate. La parte aérea de las plantas de tomates igualmente es afectada por la salinidad: las plantas alcanzan una menor altura, las hojas se presentan en menor número y a la vez manifiestan una disminución en su densidad estomática en la cara adaxial (Romero, 2001), presentan clorosis y necrosis principalmente en los bordes de las hojas. El área foliar también disminuye (Romero, 2001; Al-Karaki, 2000). Los frutos se afectan adversamente en su rendimiento (Pérez-Alfocea, 1996; Grainferberg et al., 2000; Faiz-SMA et al., 1994; Carvajal et al., 2006), pero positivamente en cuanto a algunos atributos organolépticos y/o de interés para la agroindustria, puesto que presentan un mayor contenido de compuestos solubles, sólidos totales, acidez titulable y carotenoides.

Para atenuar el efecto adverso de las sales en el rendimiento del cultivo del tomate existe una serie de prácticas agronómicas posibles de implementar, tales como el lavado de los suelos, su micronivelación, mejoramiento del drenaje (FAO, 1994), inoculación con micorrizas (Martínez et al., 2004; Al-Karaki y Hammad, 2001; Copeman et al., 1996), aplicación de una fertilización potásica (Satti-SME et al., 1994), injerto sobre patrones tolerantes (Estañ et al., 2005; Rivero et al., 2003), y uso de mulch (Munns et al., 2005), entre otras.

La presente monografía analiza los efectos de la salinidad en la germinación de semillas de tomate como también sus efectos en los diferentes órganos de las plantas, y revisa el manejo agronómico de este cultivo en condiciones salinas. Con estos tres objetivos se pretende justificar dos hipótesis: A) la salinidad altera la germinación y el crecimiento de las plantas de tomate. B) para reducir los efectos adversos de la salinidad es importante considerar un buen manejo agronómico del cultivo.

\section{ESTADO ACTUAL DEL PROBLEMA}

\section{GERMINACIÓN DE SEMILLAS}

Los efectos de la salinidad en el porcentaje de germinación de las semillas de tomate se encuentran estrechamente relacionados a las concentraciones de 
las sales en el medio de siembra, como también al cultivar o especie de que se trate. El tiempo en que tardan en germinar también se prolonga (Cuartero y Fernández-Muñoz, 1996; El-Habbasha et al. 1996; Singer-SM, 1994).

La tolerancia a la salinidad de las semillas en su germinación es una medida de la habilidad de éstas para soportar los efectos de altas concentraciones de sales solubles en el medio. La presencia de sales en el medio disminuye el potencial hídrico, provocando una menor disponibilidad de agua para las semillas, de manera que éstas deben generar suficiente potencial osmótico para mejorar el estatus hídrico de los embriones y permitir su crecimiento (Jones, 1986).

La tolerancia a salinidad en la germinación de muchas especies no está consistentemente relacionada a la tolerancia durante la emergencia, crecimiento vegetativo, floración y fructificación. Así, por ejemplo, betarraga, cebada y algodón, cultivos tipificados como de alta tolerancia a las sales, son relativamente sensibles durante la germinación y en el estado de plántula. Otras especies como el maíz, arvejas y habas son más sensibles durante estados más avanzados de desarrollo (Pessarakli, 1994). Considerando los resultados de otras investigaciones el tomate tendría un comportamiento semejante a la betarraga, cebada y algodón, puesto que al experimentar con plantas de tomates cv Daniela a las cuales se les suministró en la solución nutritiva 0 , 20,40 y $60 \mathrm{mM} \mathrm{NaCl}$ en tres estados de crecimiento (16 días después del trasplante, inicios de floración y comienzos del desarrollo del fruto) se determinó que la tolerancia a las sales incrementaba cuando la aplicación se realizaba más tardíamente, es decir, en estados más avanzados de la ontogenia de las plantas de tomates (Del Amor et al., 2001).

Estudios en los cuales trataron semillas de diversos cultivares de L. esculentum a concentraciones crecientes de $\mathrm{NaCl}$, demostraron que el porcentaje de germinación disminuye con el aumento de la salinidad, y a la vez se prolonga el período de germinación (Cuartero y Fernández-Muñoz, 1999; El-Habbasha et al., 1996; Singer-SM, 1994). Por ejemplo, se determinó que el cultivar Edkawi sal-tolerante presentaba una germinación del $90 \%$ a $85,5 \mathrm{mM}$ de $\mathrm{NaCl}$ y esta disminuía al $71,6 \%$ cuando el $\mathrm{NaCl}$ se incrementaba a $171 \mathrm{mM}$. A una concentración de 128,2 mM de $\mathrm{NaCl}$ aumentó el número de días requeridos para la germinación, en porcentajes que variaron entre 23 y 23,9\%, depen- diendo de los cultivares, en comparación con los controles (El-Habbasha et al. 1996). Otros estudios indican que las semillas de tomate requieren de un $50 \%$ de días adicionales para germinar cuando están en un medio con $80 \mathrm{mM}$ de $\mathrm{NaCl}$ en comparación con un medio sin sal, y necesitan casi el doble de días si se encuentran sometidas a $190 \mathrm{mM} \mathrm{NaCl}$ (Cuartero y Fernández-Muñoz, 1999).

Respecto a los límites máximos de tolerancia a salinidad en la germinación de Lycopersicon esculentum, también éstos son función del cultivar de que se trate. Existe una variabilidad en la respuesta de éstos. Por ejemplo, al analizarse la respuesta a estrés salino en los cultivares de L. esculentum, Marikit, Improved Pope, 95-43, y VF-134-2 en todos ellos la germinación fue inhibida a $200 \mathrm{mM}$ (Del Rosario et al., 1990). En cambio otras investigaciones en las cuales se sometieron a una alta concentración salina $(330 \mathrm{mM} \mathrm{NaCl})$ las accesiones de L. esculentum: Edkawy, Volgogradiskij, Pera, PE-64 var. Cerasiforme, Mucha miel y Mex-112 var. Cerasiforme registraron una germinación del 30, 2, 2, 2, 0, y 0\%, respectivamente (Cuartero y Fernández-Muñoz, 1999).

En cuanto a la tolerancia a salinidad entre distintas especies, investigaciones que evaluaron 20 accesiones de $L$. peruvianum, 2 accesiones de L. pimpinellifolium y 6 de L. esculentum empleando $\mathrm{NaCl}$, reportaron que tres de las veinte accesiones de $L$. peruvianum fueron más tolerantes a la salinidad, al presentar éstas una mejor germinación y crecimiento de la plúmula/ radícula hasta una $\mathrm{CE}$ de $10,2 \mathrm{dS} / \mathrm{m}$, mientras que el resto de las accesiones mostraron efectos perjudiciales, y por lo tanto una menor tolerancia, a niveles de CE superiores a 4,95 dS/m (Srinivas, 2001). Estos resultados indican la existencia de potencial genético para tolerancia a salinidad en el germoplasma silvestre de Lycopersicon.

Otras investigaciones realizadas por Cuartero y Fernández-Muñoz (1999) sobre el comportamiento de accesiones de L. esculentum, L. peruvianum y L. pennellii a concentraciones crecientes de $\mathrm{NaCl}$ indicaron que a $80 \mathrm{mM}$ disminuía algo el porcentaje de germinación, y a los $190 \mathrm{mM}$ el porcentaje de germinación se reducía drásticamente, excepto para la accesión Edkawy (L. esculentum). Sobre esta concentración de cloruro de sodio sólo cuatro de las ocho accesiones germinaron, pero lo hicieron en muy bajo porcentaje. A diferencia de los resultados obtenidos por Srinivas (2001), en esta 
investigación las accesiones más tolerantes a sales correspondieron a la especie L. esculentum y no a $L$. peruvianum, hecho que señalaría que dentro de L. esculentum también sería posible seleccionar para salinidad.

Trabajos realizados por Jones (1986) en los cuales se sometieron a $100 \mathrm{mM} \mathrm{NaCl}$ seis accesiones de L. esculentum y una accesión de L. pennellii, LA 716 , indicaron que esta última presentó una germinación del 99\%, mientras que sólo una accesión de L. esculentum tuvo igual porcentaje. En tanto que en las otras cinco accesiones la germinación varió entre un 88 y $97 \%$. Estos resultados indican que al interior de L. pennellii también existe variabilidad genética para tolerancia a salinidad.

\section{EFECTO DE LA SALINIDAD EN LAS RAÍCES}

El efecto de las sales en las raíces de las plantas de tomate siempre resulta en un menor crecimiento de estos órganos, hecho que puede afectar el crecimiento general de la planta al reducirse el volumen de suelo que pueden explorar sus raíces (Almasoum, 2000). Existe variabilidad en esta respuesta, la cual depende del cultivar o especie de que se trate, los niveles de salinidad a que son expuestas y la duración del periodo al estrés salino. Las principales sales que afectan a los vegetales y que se encuentran en los suelos corresponden a cloruros y sulfatos de sodio, calcio, magnesio y potasio (Munns et al., 2005), siendo para las plantas los principales iones citotóxicos $\mathrm{Na}^{+}, \mathrm{Cl}^{-}$y SO $\mathrm{SO}_{4}^{-}$(Chinnusamy et al., 2005).

Investigaciones en las cuales se examinó el efecto del $\mathrm{NaCl}$ sobre las raíces de los cultivares de L. esculentum: Sera, 898, y Rohaba se determinó que el aumento de la concentración de sal afectaba adversamente el crecimiento de las raíces, cuantificado como materia seca (Al-Karaki, 2000). Semejantes resultados se obtuvieron al tratar el cv de tomate (L. esculentum) [P73] sal sensitivo y la accesión silvestre de L. pennellii [PE47] con $\mathrm{NaCl}$, en ambos el desarrollo de las raíces se redujo pero fue más acentuado en P73 (Abrisqueta-JM et al., 1991).

Las sales afectan el crecimiento al alterar la absorción de agua por las raíces, fenómeno que se denomina componente osmótico, y sería el efecto inicial que padecen las plantas (Shannon y Grieve, 1999). También se desencadenan desequilibrios iónicos en las plantas por la excesiva absorción de sodio y cloruros, los que generan efectos secundarios como problemas de toxicidad y nutricionales vinculados a la absorción de iones esenciales para el crecimiento y desarrollo de las plantas (Yokoi et al., 2002).

En cuanto al componente osmótico, para superar los problemas de absorción de agua las plantas requieren acumular solutos compatibles a nivel de citosol y organelos sin afectar la actividad de las enzimas. Algunos de éstos son iones esenciales como el K+ $\mathrm{K}^{+}$, pero la mayoría son solutos orgánicos como azúcares simples (principalmente glucosa y fructosa), alcoholes derivados de azúcares (glicerol e inositoles metilados) y azúcares complejos (trialosa, resinosa y fructanos). También se incluyen derivados de aminoácidos cuaternarios (prolina, glicina betaína, $\beta$-alanina betaína, prolina betaína), aminas terciarias (1,4,5,6-tetrahidro-2 -metil 1-4 carboxil piramidina) y compuestos sulfónicos (o-sulfato de colina, propironato dimetil sulfónico) (Yokoi et al., 2002). Con ello las plantas logran disminuir el potencial osmótico, hecho que facilita el movimiento del agua hacia el interior de las células de las raíces.

En relación a la prolina, plantas de tomates (L. esculentum cv Radja) sometidas a estrés salino con niveles de $140 \mathrm{mM} \mathrm{NaCl}$ generan una acumulación significativa de prolina en el citoplasma celular de las raíces primarias y secundarias. A un estrés moderado $(70 \mathrm{mM} \mathrm{NaCl})$ sólo existen diferencias significativas de acumulación de prolina en las raíces primarias. Estos resultados sugieren que a nivel de raíces la acumulación de prolina puede ser considerada como un indicador de sensibilidad a las sales en tomate, además de contribuir como una respuesta adaptativa a la disminución del potencial osmótico en el citoplasma (Pérez-Alfocea et al., 1996).

Otro osmorregulador, la sacarosa, también cuantificada en raíces primarias del cv Radja, manifestó un comportamiento opuesto al esperado, puesto que disminuyó su concentración significativamente a niveles de $140 \mathrm{mM} \mathrm{NaCl}$. En raíces secundarias la concentración de sacarosa fue semejante a 0 y $70 \mathrm{mM} \mathrm{NaCl}$ y significativamente menor a concentraciones de $140 \mathrm{mM} \mathrm{NaCl}$. Estos resultados estarían dando cuenta que la sacarosa no es un soluto orgánico que se acumule en L. esculentum cv Radja, hecho que también reafirmaría que los solutos que acumulan las plantas varían entre las especies (Yokoi et al., 2002). 
Referente a desequilibrios iónicos y toxicidad. $\mathrm{Al}$ ingresar el sodio al citosol de las células de la raíz a través de canales de cationes o transportadores (selectivos y no selectivos) o a través de la vía apoplástica, reduce la relación $\mathrm{K}^{+} / \mathrm{Na}^{+}$en el citosol, la cual en condiciones normales debe ser alta para el buen funcionamiento celular (Chinnusamy et al., 2005). Esto resulta en niveles tóxicos de sodio y en una insuficiente concentración de potasio para algunas reacciones enzimáticas y el ajuste osmótico, dado que como se señaló anteriormente el potasio también es un soluto compatible. La toxicidad es causada por el reemplazo del $\mathrm{K}^{+}$por $\mathrm{Na}^{+}$en reacciones bioquímicas (Chinnusamy et al., 2005). Se considera también que una alta relación $\mathrm{K}^{+} / \mathrm{Na}^{+}$ mejora la tolerancia de las plantas a la salinidad (Hu y Schimdhalter, 2005).

En cuanto a los efectos nutricionales, altas concentraciones de $\mathrm{Na}^{+}$en la solución externa causan una disminución en las concentraciones de $\mathrm{K}^{+} \mathrm{y}$ $\mathrm{Ca}^{2+}$ en los tejidos de las plantas. Estas reducciones se pueden deber al antagonismo del $\mathrm{Na}^{+}$y $\mathrm{K}^{+}$ por los sitios de absorción en las raíces, el efecto del $\mathrm{Na}^{+}$en el transporte al xilema o a la inhibición de los procesos de absorción (Hu y Schimdhalter, 2005). Otros investigadores consideran que una alta concentración de $\mathrm{Na}^{+}$no sólo inhibe la absorción de nutrientes directamente por interferencia con transportadores en la membrana plasmática de la raíz, tales como los canales selectivos de $\mathrm{K}^{+}$, sino también por la inhibición del crecimiento de la raíz a causa del efecto osmótico del $\mathrm{Na}^{+}$y a los afectos adversos del $\mathrm{Na}^{+}$en la estructura del suelo (Tester y Davenport, 2003).

También se ha observado que al existir un aporte suplementario de calcio y potasio se mejora la respuesta de la planta al estrés salino. Así la adición de $\mathrm{Ca}\left(\mathrm{NO}_{3}\right)_{2}$ y $\mathrm{KNO}_{3}$ a una solución de $50 \mathrm{mM} \mathrm{NaCl}$ con la que regaron cinco cultivares de L. esculentum mejoraba el volumen radicular de éstos (López-MV y Satti, 1996).

Investigaciones en L. esculentum cv Rossel relacionada con la adición de potasio $(0,2$ y 2,0 $\mathrm{mM}$ ) en la solución salina preparada con $100 \mathrm{y}$ $200 \mathrm{mM} \mathrm{NaCl}$ reportaron que el efecto nocivo del $\mathrm{NaCl}$ disminuye al incrementarse el potasio. Cuando las plantas fueron cultivadas con $100 \mathrm{mM}$ $\mathrm{NaCl}$ y el potasio elevado de 0,2 a 2,0 mM en el medio de crecimiento, se observó una disminución significativa en la concentración de sodio, siendo éste de un $17 \%$ en las raíces. A 200 mM NaCl, la disminución en la concentración de sodio también fue significativa, siendo del orden del 19\%. Estos resultados indican que el potasio suministrado, su acumulación y la regulación que produce en el tejido de la planta contribuyen a la tolerancia de la sal y a un mejor crecimiento de las plantas de tomate (Al-Karaki, 2000).

Otros estudios que relacionaban distintos niveles de salinidad con las concentraciones de nitratos en las raíces y tallos, y con el periodo de aplicación de las soluciones salinas en tres cultivares de tomates con diferentes tolerancias a las sales, demostraron que los efectos de la salinidad en las concentraciones de nitrógeno total y particularmente nitratos dependen parcialmente de los niveles de $\mathrm{NaCl}$ y de la duración del estrés, más bien su dependencia está determinada por el grado de la tolerancia del cultivar. De los cultivares que estuvieron sometidos menos tiempo a la solución salina (tres semanas) el cv más tolerante (GC-72) mostró el mayor incremento de nitratos en las raíces. Con el aumento del periodo del estrés (10 semanas) las respuestas entre los cultivares más tolerantes fueron más similares (Pérez-Alfocea et al., 1993).

\section{DESARROLLO DE LA PARTE AÉREA: TALLOS, HOJAS, FLORES Y FRUTOS}

La altura de las plantas de tomate disminuye con el incremento de la salinidad. A nivel de hojas la salinidad genera una reducción en su número y en el área foliar (Romero-Aranda et al., 2001). También se observa clorosis, necrosis, disminución de la densidad estomática en la cara adaxial y simultáneamente un aumento en la cara abacial, aumento de clorofila e incremento de actividad de la peroxidasa (Romero-Aranda et al., 2001). También afecta la floración y la producción de frutos entre otros (Del Rosario, et al., 1990; Pérez-Alfocea et al., 1996; Cucci et al., 2000). En cuanto a su vinculación con enfermedades, favorece la incidencia de pudrición terminal en frutos de tomates.

La disminución en el número de hojas como consecuencia del incremento de la salinidad es una respuesta variable que depende de la especie o cultivar de que se trate como también de los niveles de sales a que son expuestas las plantas. Experimentos realizados en los tomates cultivados cv Daniela y Moneymarker (Romero-Aranda et al., 2001) que fueron sometidos a estrés salino con 35 y $70 \mathrm{mM} \mathrm{NaCl}$, el nivel inferior fue suficiente para 
reducir significativamente el número de hojas en el cv Daniela, no así en el cv Moneymarker, que requirió $70 \mathrm{mM} \mathrm{NaCl}$ para presentar una similar respuesta. Considerando este parámetro, se podría pensar que el cv Moneymarker es más tolerante a salinidad que el cv Daniela y que al interior de L. esculentum existe variabilidad genética para tolerancia salinidad.

El área foliar también es influenciada negativamente por la salinidad. Investigaciones sobre esta variable en los cultivares Daniela y Moneymarker señalan que el área foliar se reduce en términos significativos en ambos cultivares al exponerlos a $35 \mathrm{mM} \mathrm{NaCl}$. La disminución alcanzó un 12,3\% en el cv Daniela y un 18,5\% en Moneymarker. A $70 \mathrm{mM} \mathrm{NaCl}$ la reducción del área foliar en ambos cultivares también fue significativamente mayor, alcanzando un $16 \%$ en el cv Daniela y un 33\% en el cv Moneymarker. Por las diferencias detectadas en este último cv sugerirían su mayor sensibilidad a salinidad (Romero-Aranda et al., 2001).

La densidad estomática, al igual que la conductancia estomática son también afectadas por la salinidad. Concentraciones de $35 \mathrm{mM} \mathrm{NaCl}$ reducen significativamente la densidad estomática y la conductancia estomática en los cultivares Daniela y Moneymarker, situaciones que pueden llevar a una reducción en la absorción de agua por las plantas (Romero-Aranda et al., 2001).

El número de frutos de tomate son afectados por la salinidad. También existen diferencias en las respuestas a este estrés entre los cultivares. Investigaciones realizadas en los cv de L. esculentum Marikit, Improved Pope, VF-134-1-2 y 95-43, sometidos a un estrés salino de $150 \mathrm{mM}$ $\mathrm{NaCl}$ sólo mostraron una reducción significativa en su número en el cv VF-134-1-2, pero también se generó un aumento significativo en el caso del cv Marikit (Del Rosario et al., 1990). Estos resultados confirman que existen diferencias entre cultivares y que el cv Marikit presenta una alta tolerancia a salinidad e incluso mayor producción en condiciones salinas; también se demuestra que al interior de $L$. esculentum existe variabilidad genética para tolerancia salinidad. Al comparar la respuesta de estos cultivares con la obtenida en el tomate cultivado cv Radja (Pérez-Alfocea et al., 1996) en el cual se registró un menor número de frutos a $70 \mathrm{mM} \mathrm{NaCl}$, es posible manifestar que éste es mucho más sensible a las sales que los cultivares Improved y 95-43.
El peso de los frutos también es influenciado por la salinidad, detectándose un menor peso de los frutos al exponer las plantas del cv 95-43 a 150 $\mathrm{mM} \mathrm{NaCl}$. No ocurrió lo mismo en los cultivares Improved Pope y VF-134-1-2. En cambio, en Marikit al igual que en su número de frutos, el peso de éstos aumentó significativamente $(38,4 \%)$ al someter las plantas a estrés salino (Del Rosario et al., 1990). En experiencias semejantes realizadas en el cv Radja expuesto a 70 y $140 \mathrm{mM} \mathrm{NaCl}$ sólo se detectó un menor peso cuando las plantas se expusieron a $140 \mathrm{mM}$, de modo que este cultivar presenta un comportamiento semejante a estrés salino que el cv 95-43 (Pérez-Alfocea et al., 1996).

Vinculada a la salinidad también se encuentra la pudrición terminal de los frutos, que corresponde a un desorden fisiológico relacionado a varios factores además de la salinidad, tales como altas concentraciones de magnesio $(\mathrm{Mg})$, amonio $\left(\mathrm{NH}_{4}\right)$ y/o potasio $(\mathrm{K})$, inadecuado desarrollo del xilema, acelerada tasa de crecimiento, relaciones desfavorables de humedad (alta, baja o fluctuante), calcio poco soluble en el suelo, altas temperaturas, y alta y baja transpiración, pero la causa base de este desorden es una inadecuada cantidad de calcio en el extremo distal de los frutos (Taylor y Locassio, 2004). Otros investigadores consideran que soluciones nutrientes con una alta salinidad generalmente producen una deficiencia de calcio en los frutos debido a que ésta reduce la absorción de calcio y su distribución en la parte distal del fruto (Guichard et al., 2001). Cabe señalar que todas las variedades silvestres presentan resistencia a este desorden fisiológico (Rick, 1982).

\section{EFECTOS POSITIVOS DE LA SALINIDAD}

Al regar las plantas de tomate con aguas salobres se ha observado que algunos atributos inherentes a la calidad del los frutos mejora, por cuanto éstos presentan un mayor contenido de sólidos solubles (Del Amor et al., 2001; Fernández-García et al., 2004; Serio et al., 2004; Satti-SME y López, 1994), sólidos totales, acidez total (Guichard et al., 2001), carotenoides y licopeno (Maggio et al., 2001). Estudios sobre la respuesta de las plantas al estrés osmótico (Nichols-MA et al., 1995) muestran que a mayores niveles de estrés se mejora la calidad de los frutos, por cuanto el índice refractométrico ( ${ }^{\circ}$ Brix) registrado en éstos fue superior a los controles. Cuando se evaluaron por un grupo de panelistas, éstos fueron capaces de detectar aquellos 
frutos obtenidos de las plantas sometidas a mayores estreses, los que fueron calificados positivamente por su mejor gusto. En experiencias similares con tomates Cherry cv Gardeners Delight regados con soluciones con conductividades eléctricas de 3, 5 y $8 \mathrm{dS} / \mathrm{m}$ y sometidos a una evaluación sensorial para el gusto, un panel numeroso de jueces prefirieron los tomates tratados con una CE de $5 \mathrm{dS} / \mathrm{m}$ en el agua de riego (Gough-C y Hobson, 1990). Resultados que estarían vinculados a la relación sólidos solubles/acidez y aroma que expresan los frutos a esa $\mathrm{CE}$ del agua de riego.

Otros experimentos en los cuales se sometieron plantas de tomates cv Tombolino a riegos con aguas salinizadas con $\mathrm{NaCl}$ y $\mathrm{CaCl}_{2}$, ajustándola a $\mathrm{CE}_{\mathrm{w}}$ de 0,$5 ; 4,0 ; 8,0 ;$ y $12 \mathrm{dS} / \mathrm{m}$ prácticamente en cada una de las cuatro cosechas que se realizaron se presentaron diferencias significativas en el índice refractométrico (Cucci et al., 2000). Otro estudio en el cual se cuantificó el índice refractométrico en dos épocas del año se observó que en primavera-verano estos eran superiores $(60 \%)$ a los registrados en invierno (30\%) (Soria-T et al., 2002). Estos resultados, tal como lo expresan algunos autores (Guichard et al., 2001), se pueden deber a la exposición de los frutos durante su crecimiento a un alto déficit de presión de vapor (VPD), como es el caso de las regiones mediterráneas durante el verano, en donde los frutos presentan un significativo aumento en la concentración de azúcares respecto a los frutos expuestos a un menor VPD. Este fenómeno puede ser explicado por una disminución en la acumulación de agua en el fruto sin una significativa modificación en la cantidad de de azúcares acumulados.

Los frutos de tomates constituyen una fuente valiosa de carotenoides, en los cuales destaca el licopeno, poderoso antioxidante natural que se ha reconocido como beneficioso para prevenir enfermedades y patologías cardiovasculares. Investigaciones referentes a este pigmento en las cuales se sometieron plantas de tomates (híbrido H601) a tratamientos con aguas salinizadas detectaron que las concentraciones de carotenoides totales y licopeno se incrementaban gradualmente desde los niveles más bajos de CE $0,5 \mathrm{dS} / \mathrm{m}$ hasta los 4,4 $\mathrm{dS} / \mathrm{m}$ (aproximadamente $0,25 \% \mathrm{NaCl} \mathrm{w} / \mathrm{v}$ ), sobre este valor de conductividad comenzaba a decrecer su concentración (Maggio et al., 2001). Estos datos muestran la posibilidad de mejorar el contenido de carotenoides en los frutos con una aceptable reducción del rendimiento al regar con agua con una $\mathrm{CE}_{\mathrm{w}}$ de $4,4 \mathrm{dS} / \mathrm{m}$. No obstante estos resultados, otras investigaciones en las cuales se regaron plantas de tomates cv Naomi con una solución nutriente ajustada a 3 y $6 \mathrm{dS} / \mathrm{m}$ con $\mathrm{NaCl}$ no se detectaron efectos en el contenido de licopeno (Serio et al., 2004). Por otra parte, estudios realizados con el cv Durina $\mathrm{F}_{1}$ expuesto a CE de 2,$7 ; 4,5 ; 6,0 ; 7,5$ y 8,6 dS/m detectaron en frutos ubicados en el segundo racimo un aumento en la concentración de licopeno a 4,5 dS/m. A 6,0 $\mathrm{dS} / \mathrm{m}$ se reducía la concentración, volviendo a subir a los 7,5 dS/m, para bajar a los 8,6 dS/m (Leonardi et al., 2004). Considerando estos tres experimentos es posible afirmar que no existe una tendencia clara en la respuesta de las plantas de tomate a producir carotenoides cuando son sometidas a estrés salino. Presumiblemente en su producción, además del cv de que se trate, estén interactuando otras variables, de modo que es un tema que amerita más estudios para ser aclarado.

\section{PRÁCTICAS DE MANEJO DEL TOMATE EN CONDICIONES SALINAS}

Teniendo presente que el tomate es una hortaliza que en sus inicios se produce vía almácigos, y que es más sensible a la salinidad en la germinación y en el estado de plántula que en el estado de planta adulta, existiría la posibilidad, en los casos de contar con una restringida cantidad de agua de buena calidad, de poder cultivarlo dando los primeros riegos con esta agua.

En condiciones salinas, la fertilización necesariamente ha de considerar el aporte de potasio y calcio, puesto que el sodio, normalmente presente en los suelos salinos, interfiere en la absorción del potasio; estos iones compiten por entrar a las células de las raíces, lo cual puede significar efectos negativos en el crecimiento de las plantas. Otros investigadores (Hu y Schimdhalter, 2005) consideran que la salinidad causa una deficiencia de nutrientes en las plantas debido a la competencia tanto del $\mathrm{Na}^{+}$como del $\mathrm{Cl}^{-}$con nutrientes tales como $\mathrm{K}^{+}, \mathrm{Ca}^{2+}$ y NO $\mathrm{N}_{3}^{-}$. $\mathrm{Al}$ respecto, al evaluar plantas de tomates regadas con una solución salinizada con $50 \mathrm{mM} \mathrm{NaCl}$ se detectó que el crecimiento de hojas y tallos se reducía significativamente por la salinidad, sin embargo, cuando el potasio era adicionado el crecimiento de las plantas se optimizaba (Satti-SME et al., 1994). Igualmente el cultivo mejoraba cuando se suministraba calcio, pero en un grado inferior. También la 
adición de $\mathrm{N}$ en tomates mejora el crecimiento y rendimiento (Hu y Schimdhalter, 2005).

Vinculado a la adición de calcio está el uso de enmiendas cálcicas, es decir, la aplicación al suelo de grandes cantidades de sulfato de calcio con el fin de reemplazar los iones sodio del suelo por iones calcio. Los iones sodio liberados son luego lixiviados más allá de la zona radical usando un exceso de agua y finalmente llevados fuera del terreno mediante el drenaje (Munns et al., 2005).

El cubrimiento del suelo con residuos vegetales constituye otra práctica de manejo que atenúa el impacto de la salinidad del suelo en el cultivo, puesto que al reducirse la evaporación desde la superficie del suelo se minimiza el movimiento ascendente de las sales. Una menor evaporación también reduce la necesidad de regar, consecuentemente menos sales se acumulan en el perfil del suelo (Munns et al., 2005).

La incorporación en forma regular al suelo de materia orgánica, como rastrojos de cultivo, compost o abonos verdes, entre otros beneficios mejora la estructura del suelo y la infiltración del agua, lo cual proporciona una defensa contra los efectos adversos de las sales (Munns et al., 2005).

Otra actividad posible de incorporar en el manejo del tomate en suelos salinos es su inoculación con hongos formadores de micorrizas vesículo-arbusculares. Estos hongos pueden mejorar la producción de tomates en condiciones tanto no salinas (Martínez et al., 2004) como salinas (Al-Karaki y Hammad, 2001). La mayor productividad de plantas micorrizadas ha sido atribuida al mejoramiento en la absorción de nutrientes tales como $\mathrm{P}, \mathrm{Zn}, \mathrm{Cu}, \mathrm{K}, \mathrm{Ca}, \mathrm{Mg}$ y $\mathrm{S}, \mathrm{y}$ al mejoramiento de la absorción de agua dado que las hifas del hongo se extienden más allá de la zona donde los pelos radicales pueden alcanzar nutrientes y agua (Martínez et al., 2004).

$\mathrm{Al}$ evaluarse hongos de micorrizas (Glomus mosseae) en los cultivares de tomate Pello (saltolerante) y Marriha (sal-sensitivo) en condiciones salinas $\left(\mathrm{CE}_{\mathrm{e}} 4,9 \mathrm{dS} / \mathrm{m}\right)$ y no salinas $\left(\mathrm{CE}_{\mathrm{e}} 1,4 \mathrm{dS} / \mathrm{m}\right)$ existió a 1,4 dS/m un aumento significativo en el rendimiento por planta al inocularse éstas. A $4,9 \mathrm{dS} / \mathrm{m}$ solamente se detectaron diferencias significativas en el rendimiento en las plantas del cultivar Marriha. Al compararse el rendimiento entre las plantas inoculadas y no inoculadas cultivadas en condiciones salinas y no salinas existió un significativo mejor rendimiento en las plantas no sometidas a estrés salino $(1,4 \mathrm{dS} / \mathrm{m})$. El mejoramiento en el rendimiento por planta debido a la inoculación puede ser atribuido a un mejoramiento de la fotosíntesis asociado a un incremento en la absorción del potasio (Al-Karaki y Hammad, 2001).

Estudios anteriores a los ya citados que evaluaron la acción de hongos de micorrizas colectados de suelos salinos y suelos no salinos, en plantas de tomate cv Heinz 1350 VF 402 regadas durante 8 semanas con soluciones de $1,2,5$, y $10 \mathrm{dS} / \mathrm{m}$ (producida diluyendo $1 \mathrm{M}$ de $\mathrm{NaCl}: 1 \mathrm{M}$ de $\mathrm{CaCl}_{2}$ en agua desionizada), mostraron que el hongo proveniente del suelo salino suprimió el crecimiento de la parte aérea y raíces de las plantas (Copeman et al., 1996). Sin embargo, las plantas inoculadas con micorrizas colectadas en suelos no salinos y los testigos mostraron un incremento cuadrático en la concentración de $\mathrm{Cl}^{-}$en las hojas, en respuesta al incremento de la salinidad, mientras que las plantas inoculadas con el hongo proveniente de suelos salinos mostraron un incremento lineal en la concentración de $\mathrm{Cl}^{-}$en las hojas. De esta forma, a pesar de que el hongo originario de suelos salinos no promueve un mayor crecimiento interviene en la reducción del $\mathrm{Cl}^{-}$en las hojas a niveles moderados de salinidad, hecho que tiene implicancias positivas para la sobrevivencia de las plantas de tomate en suelos salinos.

También en el caso del tomate el injerto constituye una herramienta válida para lograr cultivar variedades de alto rendimiento, sensibles a las sales, en suelos salinos. Experiencias en el híbrido comercial de L. esculentum cv Jaguar injertado sobre cultivares con variados grados de exclusión de iones salinos tales como Radja, Pera y el híbrido Volgogradskij x Pera, expuestos a concentraciones salinas de 25,50 y $75 \mathrm{mM} \mathrm{NaCl}$, mostraron buenas respuestas en el rendimiento de frutos, sobre todo con las concentraciones de 50 y $75 \mathrm{mM} \mathrm{NaCl}$, en donde algunas combinaciones de salinidad-variedad alcanzaron un incremento en el rendimiento de frutos del $80 \%$ respecto al cultivar Jaguar injertado sobre sí mismo. Estos resultados estuvieron principalmente relacionados a las diferentes habilidades de los portainjertos para regular el transporte de iones $\left(\mathrm{Na}^{+}\right.$y Cl-) a lo largo del ciclo de crecimiento de las plantas (Estañ $e t$ $a l ., 2005)$. Las plantas injertadas más productivas mantuvieron una acumulación relativamente baja de $\mathrm{Na}^{+}$y $\mathrm{Cl}^{-}$en las hojas.

Labores culturales como cubrir la base de los tallos de las plantas (aporque) no se recomiendan 
dado que cada una de estas acciones arrastra gran cantidad de sales desde el sector superior no freático de los surcos hacia la base de los tallos de las plantas, y una vez que se riega las sales disueltas se traslocan hacia la zona radicular acentuando el efecto adverso de las sales. Referente al tipo de riego, los riegos presurizados sean estos goteo, aspersión o exudación presentan la ventaja de mantener el suelo con bajos niveles de salinidad en la zona radicular y freática (húmeda) (Chávez, 2001). Estos tipos de riegos no necesitan de aporques de modo que se evitan las acumulaciones temporales de sales en la base de las plantas.

Mantener un adecuado drenaje también es importante. Muchos problemas de salinidad encontrados en la agricultura bajo riego están asociados a niveles freáticos altos, en los cuales el agua sube a la zona radicular por capilaridad, y si el agua contiene sales, existiría una fuente continua de éstas a las raíces. La tasa de acumulación de sales en el suelo con un nivel freático poco profundo, no controlado, depende del manejo del riego, concentración de sales y profundidad del espejo del agua, tipo de suelo y condiciones climáticas. En las regiones áridas y semiáridas, problemas de salinidad generados por un deficiente drenaje no pueden ser adecuadamente controlados hasta que el nivel freático sea estabilizado y mantenido a una profundidad de por lo menos dos metros (FAO, 1994).

Lavado del suelo. Cuando la acumulación de sales en el suelo es excesiva estas pueden ser lixiviadas aplicando más agua de la requerida por los cultivos. Esta agua extra arrastraría por lo menos una porción de sales bajo la zona de las raíces. En el tiempo las sales removidas por lavado deben ser iguales o exceder a las sales adicionadas en el agua aplicada, de lo contrario las sales se acumularían pudiendo alcanzar concentraciones dañinas (FAO, 1994).

Otras prácticas importantes de considerar para atenuar el efecto de las sales son por ejemplo: la micronivelación del suelo, lo cual mejoraría la distribución del agua; empleo de métodos de riego de alta frecuencia (goteo o microjet) para evitar estrés hídrico y reducir la salinidad (Munns et al., 2002); ubicación propicia de las semillas de forma de evitar aquellas áreas probables de ser salinizadas al regar, y selección del tipo de fertilizante como su ubicación en el perfil del suelo (FAO, 1994).

\section{DISCUSIÓN}

La salinidad afecta negativamente la germinación de las semillas de tomates, sean estas las formas silvestres como las cultivadas. Estos efectos inciden en el porcentaje de germinación y el tiempo en que este proceso se lleva a cabo. A la fecha se han detectado al interior del género Lycopersicon varias especies con un grado de tolerancia a salinidad mayor que otras, incluyendo a L. esculentum, hecho importante ya que demuestra que no sólo al interior de las especies silvestres es posible detectar germoplasma con mayor tolerancia a salinidad. Estos resultados dan cuenta de la existencia de variabilidad genética en tomate y por tanto de germoplasma importante para el mejoramiento genético a estrés salino, de modo que los esfuerzos en identificar germoplasma resistente a este estrés son válidos, especialmente en aquellas especies silvestres que presenten compatibilidad genética con los cultivares comerciales como $L$. pimpinellifolium y L. pennellii.

El crecimiento de las raíces de las plantas de tomates es afectado en forma adversa por la presencia de sales en el medio de cultivo, las que reducen el potencial hídrico del agua del suelo y también generan un desequilibrio iónico que produce toxicidades y desbalances nutricionales. La intensidad de la respuesta de las plantas depende de la concentración de sales a que se exponen, de la especie o cultivar de que se trate y del tiempo de exposición al estrés. Este menor crecimiento se traduce en una disminución del largo de las raíces $y$, por tanto, también de una reducción del volumen de suelo que las raíces pueden prospectar para absorber agua y nutrientes. Para la absorción de agua las plantas de tomates acumulan solutos compatibles, los cuales no serían iguales para todos los cultivares como el caso del cv Radja, en el cual la sacarosa diminuyó su concentración al exponerse las plantas a $140 \mathrm{mM} \mathrm{NaCl}$. En los desequilibrios iónicos producidos por la salinidad uno de los principales iones responsables de estos es el sodio, el cual al ingresar al citosol produce toxicidades por el reemplazo del potasio por sodio en las reacciones bioquímicas. También hay efectos a nivel de potenciales electroquímicos de diversas membranas celulares. Igualmente, el sodio al reemplazar el calcio de las membranas celulares produce desbalances nutricionales, al afectar su integridad y selectividad para la absorción del potasio. Para 
reducir estos efectos negativos en las plantas es del todo necesario el aporte de nutrientes que contengan potasio y calcio.

Todos los órganos de la parte aérea de las plantas de tomates se alteran con la salinidad. Los tallos alcanzan menor altura, las hojas disminuyen en número, el área foliar igualmente se reduce y en los frutos se registra un menor número y peso. $\mathrm{Al}$ analizarse estos parámetros en algunos cultivares de L. esculentum se detectó una gran heterogeneidad en la respuesta a este estrés, puesto que algunos cultivares fueron extremadamente sensibles y otros más tolerantes, incluso uno presentó un mejor comportamiento en la producción de frutos en estado de estrés. Estos últimos resultados estarían señalando que algunos cultivares son fuentes de genes de gran importancia para el mejoramiento genético de esta hortaliza.

El riego con aguas salobres puede tener efectos positivos en los frutos de tomate, esto es, un aumento de los sólidos solubles, sólidos totales, carotenoides y acidez. Algunos de estos factores, como los sólidos solubles y carotenoides, al encontrarse en mayores niveles constituyen un atributo de interés o deseable para la agroindustria procesadora de esta hortaliza, dado que se reducirían costos de producción y a la vez la calidad de los productos se optimizaría. Cabe señalar que la liberación del licopeno (carotenoide) se mejora cuando el fruto es sometido a un tratamiento térmico.

\section{BIBLIOGRAFÍA}

ABRISQUETA-JM; HERNÁN-SÁEZ-A; ALARCÓN-JJ; LOZANO-MA. 1991. Root growth dynamics of two tomato genotypes under saline conditions. Suelo-y-Planta. 1(3): 351-361.

AL-KARAKI, G. N. 2000. Growth, sodium, and potassium uptake and translocation in salt stressed tomato. J-plant-nutr. Monticello, N.Y. Marcel Dekker Inc. 23 (3): 369-379.

AL-KARAKI, G. N.; HAMMAD, R. 2001. Mycorrhizal influence on fruit yield and mineral content of tomato grown under salt stress. Journal of Plant Nutrition. 24 (8):1311-1323.

ALMASOUM, A. A. 2000. Effect of planting depth on growth and productivity of tomatoes using drip irrigation with semi saline water. Acta Hort. 537: 773-778.

CARVAJAL, M.; CERDA, A.; MARTÍNEZ, V. 2000. Modification of the response of saline stress tomato plants by the correction of cations disorders. Plant-Growth-Regul. Dordrecht: Kluwer Academic Publishers. 30(1): 37- 47.

CHÁVEZ, R. A. 2001. Mejoramiento genético de plantas tuberíferas para zonas árido-salinas. Ed. Ari-graphics. Perú. 327 p.

\section{CONCLUSIONES}

La salinidad produce efectos positivos y negativos en las plantas de tomates, sean estas cultivadas o silvestres. La mayoría de los efectos son negativos, y se hacen sentir desde los primeros estados fenológicos de la planta. La germinación se reduce y se prolonga el tiempo de este evento. El crecimiento de las raíces disminuye y por tanto cae la capacidad de absorción de agua y nutrimentos. En la parte aérea el número de frutos y su peso también son alterados adversamente. El grado de impacto de las sales en el cultivo depende del estado de desarrollo en que se encuentre el cultivo, siendo las etapas juveniles más sensibles que las etapas adultas.

Existe variabilidad genética para tolerancia a salinidad a nivel de especies cultivadas y silvestres, hecho que da lugar a la realización de investigaciones en la detección de genes vinculados a este estrés.

Efectos positivos de la salinidad en las plantas de tomates cultivadas son el mejoramiento de la calidad organoléptica y biológica de los frutos, por cuanto estos presentan un mayor contenido de sólidos solubles, acidez y pigmentos carotenoides.

Para llevar en buenos términos el cultivo del tomate en regiones con problemas de salinidad es necesario, además de utilizar los cv de mayor tolerancia, incorporar en su manejo algunas prácticas que conlleven un menor grado de incidencia de las sales en las plantas.
CHINNUSAMY, VISWANATHAN; JAGENDORF, ANDRÉ; ZHU, JIAN-KANG. 2005. Understanding and improving salt tolerance in plants. Crop Science. 45: 437-448.

COPEMAN, R. H.; MARTIN, C. A.; STUTZ, J. C. 1996. Tomato grown in response to salinity and mycorrhizal fungi from saline or non saline soils. HortSience. 31 (3): 341-344.

CUARTERO J.; FERNÁNDEZ-MUÑOZ R. 1999. Tomato and salinity. Scientia Horticulture.78: 83-125.

CUCCI, G.; CANTORE, V.; BOARI, F.; DE CARO, A. 2000. Water salinity and influence of SAR on yield and quality parameters in tomato. Acta Hort. 537: 663-670.

DEL AMOR, F. M.; MARTÍNEZ, V.; CERDA, A. 2001. Salt tolerance of tomato plants as affected by stage of plant development. HortScience. 36 (7):1260-1263.

DEL ROSARIO, D. A.; SUMAGUE, A. C.; ROXAS, V. P.; BAUTISTA, T. S. 1990. Response of tomato (Lycopersicon esculentum Mill.) to salt stress. The Philippine agriculturist. 73 (2): 193-198. 
EL-HABBASHA-KM; SHAHEEN-AM; RIZK-FA. 1996. Germination of some tomato cultivars as affected by salinity stress condition. Egyptian-Journal-of-Horticulture. 23 (2): 179-190.

ESTAÑ, M.T.; MARTÍNEZ-RODRÍGUEZ, M. M.; PÉREZALFOCEA, F.; FLOWERS, T. J.; BOLARIN, M. C. 2005. Grafting raises the salt tolerance of tomato through limiting the transport of sodium and chloride to the shoot. Journal of experimental botany. 56 (412): 703-712.

FAIZ-SMA; ULLAH-SM; HUSSAIN-AKMA; KAMALATMM;ARDUS-SATTAR. 1994. Yield, mineral contents and quality of tomato (Lycopersicon esculentum) under salt stress in a saline soil. Current-Agriculture. 18 (1-2): 9-12.

FAO. 1994. Water quality for agriculture. 29 Rev. 1.

FAO. 2000. Global network on integrated soil management for sustaintable use of salt-affected soils. Rome, Italy: FAO Land and Plant Nutrition Management.

FERNÁNDEZ-GARCÍA, N.; MARTÍNEZ, V.; CERDA,A.; CARVAJAL, M. 2004. Fruit quality of grafted tomato plants grown under saline conditions. Journal of Horticultural Science and Biotechnology. 79 (6): 995-1001.

FOOLAD, M.R.; LIN, G.Y. 1997. Genetic potential for salt tolerance during germination in Lycopersicon species. HortScience. 32 (2): 296-300.

GOUGH-C; HOBSON-GE. 1990. A comparison of the productivity, quality, shelf-life characteristics and consumer reaction to the crop from cherry tomato plants grown at different levels of salinity. Journal-of-Horticultural-Science. 65: (4) 431-439.

GRAINFERBERG-A; GIUSTINIANI-L; BARSANTI-L; BOTRINI-L. 2000. Effect of salt-stress on tomato fruit quality. Colture-Protette. 29 (6): 71-80.

GUICHARD, SORAYA; BERTIN, NADIA; LEONARDI, CHERUBINO; GARY, CHRISTIAN. 2001. Tomato fruit quality in relation to water and carbon fluxes. Agronomie. 21: 385-392.

HU, YUNCAI; SCHIMDHALTER, U. 2005. Drought and salinity: A comparison of their effects on mineral nutrition of plants. J. Plant Nutr. Soil Sci. 168: 541-549.

JONES, R.A. 1986. High salt tolerance potential in Lycopersicon species during germination. Euphytica; 35: 575-582.

LEONARDI, M. M.; GIUSSRIDA, S.; FOGLIANO, V.; PERNICE, R. 2004. TOMATO fruit quality in relation to the content of sodium chloride in the nutrient solutions. Università di Napoli. Italy. 7 p.

LÓPEZ-MV; SATTI SME. 1996. Calcium and potassium enhanced growth and yield of tomato under sodium chloride stress. Plant-Science-Limerick. 114 (1):19-27.

MAGGIO, A.; FOGLIANO, V.; AMBROSINO, P.; RITIENI, A.; DE PASCALE, S. 2001. Irrigation with saline water improves carotenoids content and antioxidant activity of tomato. Journal of Horticultural Science and Biotechnology. 76 (4): 447-453.

MARTÍNEZ, F. M.; REYES, J. P.; DESPAIGNE, F. H.; BALDAQUíN, M. H. 2004. Efecto de los hongos micorrisógenos arbusculares en el crecimiento y desarrollo del cultivo del tomate. Universidad de Granma. Facultad de Ciencias Agrícolas. Cuba. $6 \mathrm{p}$.

MUNNS, RANA. 2005. Genes and salt tolerance: ringing them together. New Phytologist. 167 (3): 645-660.
MUNNS, RANA; GOYAL, SHAM S.; PASSIOURA, JOHN. 2005. Salinity stress and its mitigation. University of California, Davis. 19 p.

MUNNS, R.; HUSAIN, S.; RIVELLI, A. R.; JAMES, R. A.; CONDON, A. G.; LINDSAY, M. P.; LAGUDAH, E. S.; SCHAHTMAN, D. P.; HARE, R. A. 2002. Avenues for increasing salt tolerance of crops, and the role of physiologically based selection traits. Plant and Soil. 247 (1): 93-105.

NICHOLS-MA; FADALLAN-EF; FISHER-KJ; MORGANLM; GERASOPOULOS-D (ED.); OLYMPIOS-CH (ED.); PASSAM-H. 1995. The effect of osmotic stress on the yield and quality of tomatoes. Acta-Horticulturae. 379: 105-111.

NUEZ FERNANDO. 2001. El cultivo del tomate. Ed. MundiPrensa. $793 \mathrm{p}$

PÉREZ-ALFOCEA-F; BALIBREA-ME; SANTA-CRUZ-A; ESTAN-MT. 1996. Agronomical and physiological characterization of salinity tolerance in a commercial tomato hybrid. Plant-and-Soil. 180 (2): 251-257.

PÉREZ-ALFOCEA-F; ESTAN-MT; SANTA-CRUZ-A; BOLARIN-MC. 1993. Effects of salinity on nitrate, total nitrogen, soluble protein and free amino acid levels in tomato plants. Journal-of-Horticultural-Science. 68 (6): 1021-1027.

PESSARAKLI, M. 1994. Handbook of plant and crop stress. University of Arizona. Tucson. Arizona. 697 p.

RICK, C. M. 1982. The potential of exotic germplasm for tomato improvement. Plant improvement and somatic cell genetics. p. 1-28.

RIVERO, R. M.; RUIZ, J. M.; ROMERO, L. 2003. Role of grafting in horticultural plants under stress conditions. Food, Agriculture \& Environment. 1 (1): 70-74.

ROMERO-ARANDA, R.; SORIA, T.; CUARTERO, J. 2001. Tomato plant-water uptake and plant-water relationships under saline growth conditions. Plant Science. 160: 265-272.

SATTI-SME; IBRAHIM-AA; AL-KINDI-SM. 1994. Enhancement of salinity tolerance in tomato: implications of potassium and calcium in flowering and yield. Communications-in-Soil-Science-and-Plant-Analysis. 25 (15-16): 2825-2840.

SATTI-SME; LÓPEZ, M. 1994. Effect of increasing potassium levels for alleviating sodium chloride stress on the growth and yield of tomato. Commun. Soil Sci. Plant Anal. 25 (15-16): 2807-2823.

SATTI-SME; LÓPEZ, M; Y FAHAD, A. AL-SAID. 1994. Salinity induced changes in vegetative and reproductive growth in tomato. Commun. Soil Sci. Plant Anal. 25 (5-6): 501-510.

SERIO, FRANCESCO; DE GARA, LAURA; CARETTO, SOFÍA; LEO LUCÍA; SANTAMARÍA, PIETRO. 2004. Influence of an increased $\mathrm{NaCl}$ concentration on yield and quality of cherry tomato grown in posidona (Posidonia oceanica (L) Delile). 14: 1885-1890.

SHANNON, M. C.; GRIEVE, C. M. 1999. Tolerance of vegetable crops to salinity. Scientia Hortoculturae. 78: 5-38.

SINGER-SM. 1994. Germination responses of some tomato genotypes as affected by salinity and temperature stress. Egyptian-Journal-of-Horticulture. 21 (1): 47-64.

SINGH, K. N.; CHATRATH, R. 2001. Breeding for adaptation to environmental factors. Chapter 8. Salinity Tolerance. $170 \mathrm{p}$. 
SORIA-T; CUARTERO-J; ROMERO-ARANDA-R; AKSOY-U (ED.); ANAC-D (ED.); ANAC-S (ED.); BELTRAO-J(ED.); BEN-ASHER-J (ED.); CUARTERO-J (ED.); FLOWERS-TJ (ED.); HEPAKSOY-S. 2002. Yield and fruit quality of salinised tomato plants with enhanced Ca fertilization. Acta-Horticulturae. 573: 35-41.

SRINIVAS, T.R. 2001. Salinity tolerance of tomato germplasm during germination. Seed Science and Technology. 29 (3): 673-677.

TANWAR, B. S. 2003. Saline water management for irrigation. International Commission on irrigation and drainage. New Delhi, India. $140 \mathrm{p}$.
TAYLOR, MATTEW, D.; LOCASSIO, SALVADORE J. 2004. Blossom-end rot: A calcium deficiency. Journal of Plant Nutrition. 27 (1): 123-139.

TESTER, MARK; DAVENPORT, ROMOLA. 2003. $\mathrm{Na}^{+}$ tolerance and $\mathrm{Na}^{+}$transport in higher plants. Annals of Botany. 91: 503-527.

USDA. U.S. DEPARTMENT OF AGRICULTURE. E.R.S. 2003. Economic Research Service. U.S. Tomato Statistics (92010). Mar

YOKOI, SHUJI; BRESSAN RAY, A.; MIKE HASEGAWA, P. 2002. Salt stress tolerance of plants. JIRCAS Working Report 25-33. 